\title{
Design of a Child Localization System on RFID and Wireless Sensor Networks
}

\author{
Chao Chen \\ Department of Engineering, Indiana University_Purdue University Fort Wayne, Fort Wayne, IN 46805, USA \\ Correspondence should be addressed to Chao Chen, chen@engr.ipfw.edu
}

Received 29 July 2009; Revised 20 November 2009; Accepted 24 February 2010

Academic Editor: Weilian Su

Copyright $\odot 2010$ Chao Chen. This is an open access article distributed under the Creative Commons Attribution License, which permits unrestricted use, distribution, and reproduction in any medium, provided the original work is properly cited.

Radio Frequency Identification (RFID) and wireless sensor networks are wireless technologies that rapidly emerge and show great potential. Combining RFID and wireless sensor networks provides a cost-efficient way to expand the RFID system's range and to enable an RFID system in areas without a network infrastructure. These two technologies are employed to build a wireless localization system in a children's theme park. The main purpose of this child localization system is to track and locate children within a certain range near some landmarks in the park. The design experience in this project can be exported to other applications such as object tracking and surveillance.

\section{Introduction}

In recent years, Radio Frequency Identification (RFID) technology has emerged to be a popular replacement for the Universal Product Code (UPC) barcode system in many industries. Specifically, RFID uses a short-range radio technology to communicate mainly digital information between a stationary location and a movable object or between movable objects. Since RFID does not require line-of-sight communication and offers a longer operating range, it outperforms barcode systems in many areas, such as electronic road tolling, supply chain management, and article tracking [1]. An RFID system generally consists of simple devices called tags (or transponders) and more complex devices called readers (or interrogators). RFID tags are small and inexpensive and can be deployed economically in very large numbers. Moreover, RFID tags usually carry a unique identity (UID) and can be attached to the objects to be managed. RFID readers employ tag-reading algorithms that are capable of identifying tags by the UIDs. Compared with the tags, the readers are more capable and are usually connected to a host computer or a network.

A wireless sensor network consists of many spatially distributed devices called motes. These devices use sensors to monitor conditions such as temperature, sound, vibration, pressure, motion, or pollutants. Since motes are small and inexpensive, they can be produced and deployed in large numbers. Motes are equipped with wireless communication capability to other motes either directly or via multiple hops. The fast-growing research effort in academia and industry has resulted in many protocols and applications of wireless sensor networks [2].

Combining RFID devices with wireless sensor networks offers advantages in both sides. First, attaching the RFID readers enables the motes to monitor a wider range of objects than with traditional sensors. Second, the wireless sensor network can expand the RFID system's range and also enable an RFID system in areas where a network infrastructure (e.g., Internet) does not exist. In this undergraduate student design project, we employ both RFID and wireless sensor network technologies to build a wireless localization system in a children's theme park. Our system design supports real-time detection of RFID tags and remote data collection through the underlying wireless sensor network. These capabilities are implemented with very low power consumption.

The rest of the paper is organized as follows. Some related projects that integrate RFID and wireless sensor networks are described in Section 2. The system requirements of the children's theme park and the detailed design of the wireless localization system are discussed in Section 3. The testing of the designed system prototype is included in Section 4. Finally, Section 5 concludes this paper. 


\section{Related Projects}

In this section, we describe several related projects and products that are taken places in industrial and academic research laboratories as well as those implemented in realworld applications.

2.1. Hands-On RFID. Intel Research Seattle has developed hand-worn RFID readers for detecting intentional touches of objects [3]. These RFID readers can be embedded in wearable gloves and bracelets and detect tagged objects through user interaction. The readers are connected to sensor motes and report sensed events wirelessly to a PC base station that may be 15 to 30 meters away. The integration of the RFID and the wireless sensor networks serves to support realtime analysis of data streams and to keep the wearable device small and autonomous. The designed hands-on RFID system can be applied to activity-based applications such as health monitoring, factory-floor maintenance, and contextsensitive reminders.

2.2. Ragobot: Networked Gaming Robots. The Networked and Embedded Systems Laboratory in UCLA has designed coordinated "real action gaming robots" (Ragobots) to form a mobile sensor network. Ragobots are teamed up in "robogaming", where they must collaborate to achieve a final goal while navigating a terrain. Specifically, Ragobots use an RFID system to provide a cost-efficient solution to object recognition. All the objects on the game board are tagged and classified. Small RFID readers mounted on the Ragobots can detect the tags and determine the object type based on the information stored on the tag [4]. Moreover, the RFID system in Ragobots adapts the mobile sensor motes to the environment and task dynamics.

2.3. WiFi Kid Tracker. In 2004, Bluesoft, a company building WiFi-based wireless security and location solutions, and KidSpotter, a location based services company, successfully deployed a full-scale child-tracking application within LEGOLAND Denmark, one of the Europe's largest amusement parks [5]. This kid tracking solution is based on Bluesoft's AeroScout System-a real-time location system that can accurately locate both standard WiFi devices and AeroScout's WiFi-based active RFID tags. Through this system, park guests can rent the AeroScout WiFi Tags with a wristband that children wear inside the park. If a child gets separated, the parents simply send a text message (i.e., SMS) from their mobile phone and receive an automated response telling them the accurate location of the child. This project motivated us to combine the RFID technology with the wireless sensor network technology for a tracking application in a smaller-scale children's park.

2.4. Patient Monitoring and Localization. IMEC-Netherland (IMEC-NL), a Dutch research institute, has built prototypes of a human body-monitoring system using $2.45 \mathrm{GHz}$ active RFID tags integrated with sensors to record and transmit data about a patient's vital signs to a central system [6].
Currently, a hospitalized patient suffering from epilepsy and apnea is monitored via electrodes with wires in a box that is attached to his face. IMEC-NL has proposed to use wireless sensors to replace the box and monitor the patient's brain activity, providing patients with mobility and perhaps even allowing them to be monitored in their homes. If the sensors detect unexpected brain activities, they transmit an alarm to an RF interrogator 10 meters away.

University Hospital of Ghent in Belgium has implemented a similar RFID-based real-time locating system to track a patient's location in the event of an emergency [7]. The integrated RFID-sensor network detects when a patient is having cardiac distress and sends to the caregivers an alert indicating the patient's location. The system uses AeroScout T2 active Wi-Fi tags, which transmit $2.4 \mathrm{GHz}$ signals carrying the tags' UIDs to the hospital's Wi-Fi network.

2.5. Disaster Relief. Telepathx, a wireless and communications company based in Melbourne, Australia, has introduced an RFID-based sensor designed to alert firefighters within minutes of a fire's ignition [8]. The proposed scheme integrates an active RFID tag and wireless thermal sensors. When the sensors discern temperatures within 2 degrees of a predetermined setting, they activate the RFID tag, communicating its UID to a reader. A notification is then sent to a person's cell phone to notify the firefighters to respond quickly and efficiently.

The Japanese Ministry of Internal Affairs and Communications (MIC) has also developed a system that allows for detailed information gathering about a disaster area by sprinkling RFID sensor tags from the sky [9]. The RFID tags are used to collect important information including the possible existence of human disaster victims and send out the gathered data through a mesh-like wireless network.

2.6. Assets Monitoring and Tracking. There are numerous other real-world scenarios of integrating RFID and wireless sensor networks in assets monitoring and tracking. Examples include US Navy's wireless RFID system to monitor the condition of valuable aircraft parts in storage [10], Siemens IT Solutions and Services' cargo-tracking system that combines RFID, wireless sensors, and GSM and satellite services [11], ZigBeef's long-range RFID system that helps ranchers and rodeos track animals from a distance [12], and so forth.

\section{System Design}

The detailed description of our design and implementation of the project is covered in this section. We start with system requirements and an overview of the child localization system and then describe system components and design considerations. We present the overall system architecture and the characteristics of our child localization system. Other possible design choices are also discussed.

3.1. System Overview. Indiana University-Purdue University Fort Wayne (IPFW) has proposed to build a children's theme park on campus at a location without any existing 
wired/wireless network coverage. There will be about fourteen statues of mastodons (i.e., the official mascot of IPFW) located in the park. The distance between any two nearby mastodons is expected to be around 10 to 30 meters. Our task is to embed the mastodon park with a high-tech architecture on which some interactive games for kids can be easily added [13].

Based on the requirements, we propose an underlying wireless localization system. The main purpose of such a system is to enable tracking and locating a child within a certain range (2-3 meters) near some landmarks. Here in this theme park, the landmarks are the statues of mastodons. Each child playing in the park will wear an RFID tag. When a child is near a mastodon, the RFID reader on or close to the statue will detect the tag and recognize it through the tag's UID. This information can be propagated through the underlying sensor network by wireless communication. One sensor mote can act as a gateway and is connected to a host computer through either wireless or wire-line connection. Therefore, the host computer can track the whereabouts of every child in the park and display the information. Furthermore, some interactive activities can be applied to the park based on this localization system. For example, some display about a mastodon can be activated when a child is close, and a child can have a print-out of his/her experience in the park.

3.2. System Components. The main hardware components of the child localization system are RFID tags and readers, a wireless sensor network, and motion detection sensors. The purpose of adding motion detection sensors is to save energy when no human activity is detected in the mastodon park.

3.2.1. RFID System. RFID tags are classified by the energy source as passive and active tags. A passive RFID tag has no power supply of its own. Thus, passive RFID tags have to be in the close range of a reader and make use of the incoming radio waves broadcast by the reader to power the response. On the other hand, an active RFID tag uses its own battery power to perform all operations. Therefore, active RFID tags usually produce a longer read range and achieve higher efficiency.

In our child localization system, we use passive RFID tags for the children to wear. Since passive RFID tags are dormant unless powered by the energy radiated by the reader when they are close, the tags pose no harm to the children. Although the operating distance is limited to the reader's range, this is not a disadvantage of our system: we want to draw children close to the mastodons to make the upper-level applications more interactive. Moreover, passive tags do not need battery replacement and thus have a low cost. The cost of a passive RFID tag usually varies from 50 cents to about 2 dollars.

RFID readers generally fall into two categories-high frequency (HF) and ultra-high frequency (UHF). HFbased RFID-systems often use $13.56 \mathrm{MHz}$ frequency, whereas UHF-based RFID systems use higher frequency ranges around $860 \mathrm{MHz}$ to $960 \mathrm{MHz}$. Besides the operating frequency, $\mathrm{HF}$ and UHF readers also differ in the read range, the

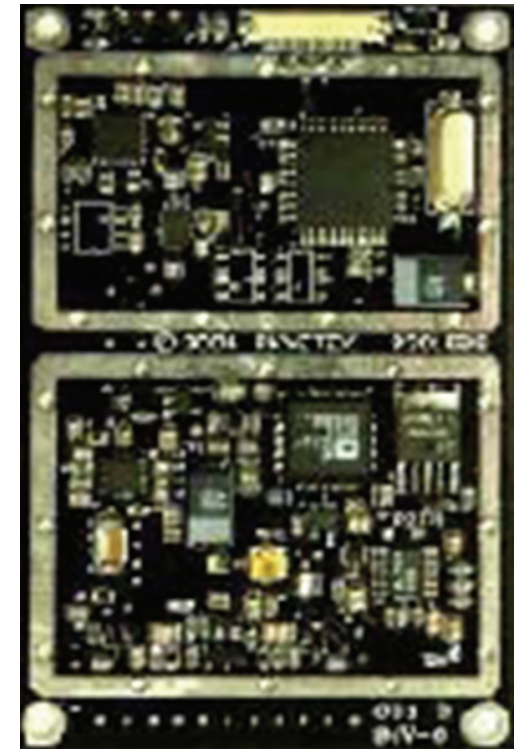

Figure 1: SkyeTek M8 RFID UHF reader [16].

read rate, the memory size, and the power source $[14,15]$. We select UHF RFID readers mainly because they have a longer read range ( $>1$ meter). Moreover, UHF RFID readers have a faster reading speed and a larger memory size.

Our system uses the SkyeTek M8 UHF $900 \mathrm{MHz}$ reader [16] as the RFID readers. It supports multiple types of UHF tags such as ISO18000-6A/B and EPC Class 0/0+ and Class 1 tags. The SkyeTek M8 has a 20-pin flex cable connector, from which a host system can supply power and communication. Three types of microcontroller host interfaces (UART, $\mathrm{I}^{2} \mathrm{C}$, and SPI) are supported for the ease of integration into existing systems. Through any of the supported host interfaces, the M8 reader operates under host control via the SkyeTek Protocol [17]. In addition, the M8 reader has a small form of $5.1 \times 7.6 \times 0.6 \mathrm{~cm}$ (see Figure 1 ) and consumes low power of $250 \mathrm{~mA}-600 \mathrm{~mA}$ in the active mode (refer to Table 1). The M8 reader is equipped with an internal antenna with a read range of around 1 meter. Moreover, an external antenna can be installed to extend the range as far as 2 meters. The marked tag read rate is 50 tags per second, which is sufficient for our child localization system.

3.2.2. Wireless Sensor Network. The main purpose of the wireless sensor network is to gather data in each reader and relay the data to a PC base station located in farther distance. The sensor mote that we use is Crossbow's Mica2 410 mote platform [18], which is a $433 \mathrm{MHz}$ radio transceiver including an ATMega128L microcontroller, an RF transceiver, and an RF antenna. The ATMega128L has a $128 \mathrm{kB}$ flash memory and a $4 \mathrm{kB}$ EEPROM, supporting programmable UART and $\mathrm{I}^{2} \mathrm{C}$ interfaces, as well as a $38.4 \mathrm{kbps} R F$ data rate. The $\mathrm{I}^{2} \mathrm{C}$ interface enables Mics2 410 sensor motes to connect directly to the SkyeTek M8 RFID reader. The Mica2 410 mote is very small in size $(5.7 \times 3.2 \times 0.7 \mathrm{~cm})$ and is shown in Figure $2(\mathrm{a})$. The mote uses two AA batteries and consumes very little power in the active mode. It also supports a power-down 
TABLE 1: Power consumption of hardware components in the client node.

\begin{tabular}{lll}
\hline & Power supply & Current \\
\hline \multirow{2}{*}{ SkyeTek M8 reader } & $5 \mathrm{~V}$ & $600 \mathrm{~mA} @ 500 \mathrm{~mW}$ output \\
& $250 \mathrm{~mA} @ 15 \mathrm{~mW}$ output \\
& $<50 \mu \mathrm{A}$ sleep \\
\hline Crossbow Mica2 mote & $3 \mathrm{~V}$ & Processor: $12 \mathrm{~mA} @$ full operation, $10 \mu \mathrm{A}$ sleep \\
& & Radio: $7 \mathrm{~mA}$ receive, $10 \mathrm{~mA}$ transmit $(1 \mathrm{~mW}$ power $), 1 \mu \mathrm{A}$ sleep \\
& Serial flash memory: $15 \mathrm{~mA}$ write, $4 \mathrm{~mA}$ read, $3 \mu \mathrm{A}$ sleep \\
\hline
\end{tabular}

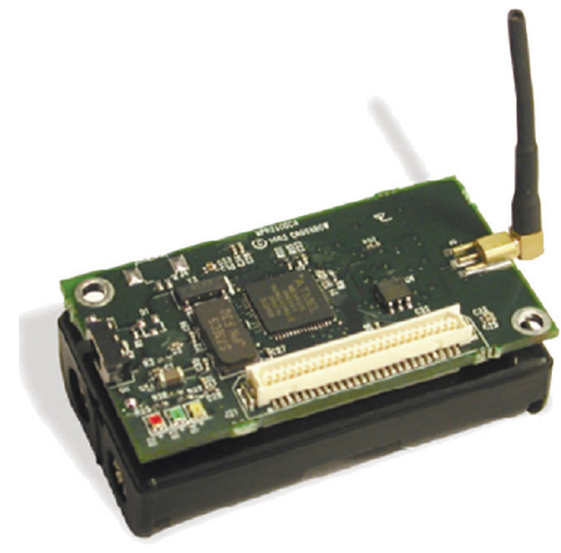

(a) Mica2 mote processor and radio platform [18]

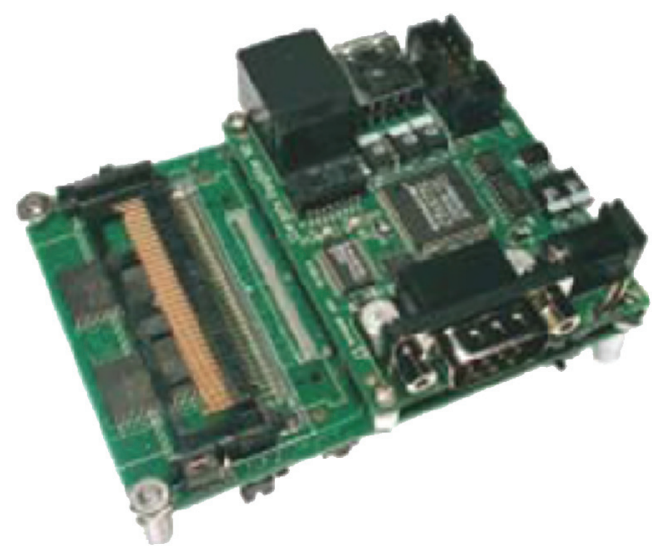

(b) Stargate gateway SPB 400 [19]

FIgURE 2: Crossbow wireless sensor network devices.

mode, which can be used to conserve power consumption when no child is near any mastodon. (Please refer to Table 1 for the current requirements of Mica2 410 mote). Moreover, a Mica2 410 sensor mote can report sensed events either directly or multihop to a Crossbow's Stargate gateway SPB 400 via a wireless channel to a Crossbow's Stargate gateway SPB 400 [19]. The Mica2 mote implements such communication through a 51-pin connector that can be connected to the Stargate gateway as a programming interface. The Stargate gateway (see Figure 2(b)) has both a standard RS-232 serial port and an AmbiCom Wave2Net wireless $802.11 \mathrm{a} / \mathrm{b}$ card, which can transfer signals to a PC base station through either wired or wireless connection.

3.2.3. Motion Detection Sensors. The main purpose of including motion detection sensors in the design is to conserve energy. When there is no human activity, thus no motion, in the park, the RFID readers are in the dormant state. Whenever motion is detected close to a mastodon, the motion detection sensor will activate the RFID reader. It is important that the read range of the RFID reader be shorter than that of the detection range of the sensor, so that there will be a short interval after the RFID reader is enabled and before it is ready for tag reading. We select the AMB315920 sensor [20] manufactured by Panasonic in our child localization system. The AMB315920 is an area-reflective-type motion sensor and has a detection range of 2 meters.
3.2.4. Software Components. The Crossbow sensor motes are operated by the TinyOS system, which is an opensource event-driven operating system designed specifically for wireless embedded sensor networks. TinyOS is written in nes $\mathrm{C}$, a language supported by many microcontrollers, and includes the necessary features to interface with hardware. In addition, the SkyeTek M8 RFID reader can communicate with any host software that sends commands according to the SkyeTek Protocol [17].

3.3. System Architecture. Figure 3 shows the system architecture of the child localization system. In this system, there are two types of nodes: a server gateway and several client nodes (one for each mastodon). The server gateway connects to a host computer and also has an RF antenna to communicate with the client nodes. A client node has the following components: a sensor mote, an RFID reader, a motion detection sensor, and a battery supply. The detailed schematics for the client node and the server gateway are shown in Figure 4.

The main characteristics of our child localization system are summarized as follows.

(i) Real-time detection: Whenever a child (with a tag) is within an RFID reader's read range, the reader can detect the child instantly and retrieve the UID from the tag. The RFID reader will also send the UID to the sensor mote that it is connected to via 


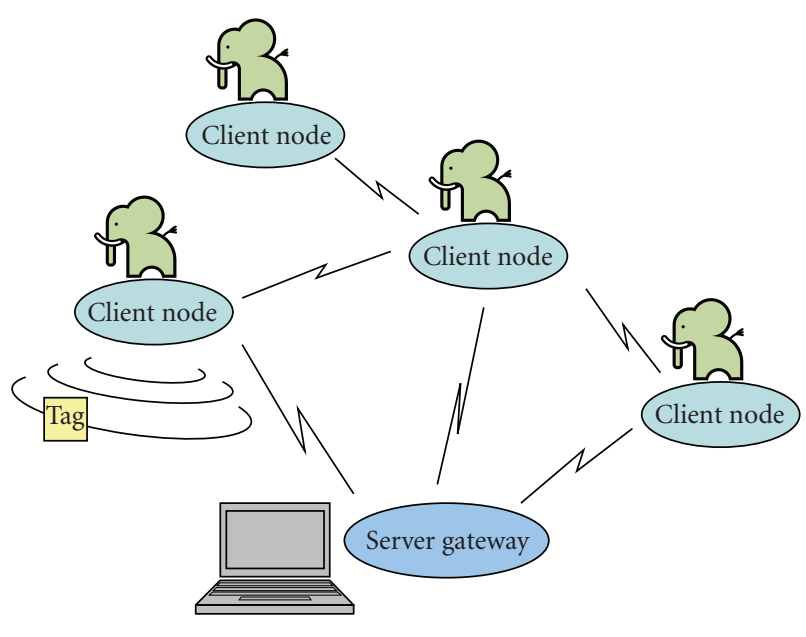

FIgURE 3: System architecture.

the $\mathrm{I}^{2} \mathrm{C}$ interface. The communication through the $\mathrm{I}^{2} \mathrm{C}$ interface is guaranteed for a data rate up to $400 \mathrm{kbps}$. The TinyOS operating system in the sensor mote handles hardware events asynchronously, thus supporting real-time data fetching from the RFID reader. Moreover, anticollision algorithms can be implemented to enable a single reader to read multiple tags in the reader's field.

(i) Remote data collection. With the help of the wireless sensor network, the detected UID information can be sent to the server gateway. A client node has the capability to communicate with the sensor mote in the server gateway. If the client node is out of range of the server gateway, some other client nodes can forward its data to the gateway. Furthermore, the Crossbow Stargate gateway board accesses the PC base station with a high speed via the wireless 802.11 card. Therefore, the PC base station can monitor the RFID detection in the park remotely.

(ii) Power conservation. All components in the client nodes are built to consume low energy. For example, operating at $5 \mathrm{~V}$, the SkyeTek M8 reader draws a current of $250 \mathrm{~mA}$ to $600 \mathrm{~mA}$ in the active mode and as low as $50 \mu \mathrm{A}$ in the sleep mode, making it appropriate for use in battery operated applications. The Crossbow Mica2 mote uses two AA batteries and draws a very low current in the active mode and about $14 \mu \mathrm{A}(10 \mu \mathrm{A}$ for processor $+1 \mu \mathrm{A}$ for radio $+3 \mu \mathrm{A}$ for serial flash memory, as shown in Table 1) in the sleep mode. The details of the power consumption of the hardware components in the client node are listed in Table 1. Moreover, the motion detection sensor can further reduce the power consumption when no motion is detected in its range.

In summary, our system design integrates motion detection sensors, RFID readers, and the wireless sensor network together to implement a cost and power efficient real-time child localization system. Compared with the WiFi Kid
Tracker system in Section 2, our design is different at least in the following aspects. First, the operation of the Kid Tracker system relies on an existing wireless network infrastructure. Our child localization system is set up on a wireless sensor network, which can be deployed easily in an area without any wired/wireless network infrastructure. Second, the Kid Tracker system locates the accurate position of a child inside the park. The purpose is to help parents find a lost child. On the other hand, our system detects a child when he/she gets closer to a landmark, so that some interactive games can be activated to attract the kid and such interaction can be reported to a central server. The goal is to enhance children's experience as they explore the theme park. Furthermore, in order to retrieve the accurate position of an RFID tag, the Kid Tracker system needs to implement some localization algorithm and collect information such as the distances of the RFID tag to several WiFi access points. In our child theme park, since the precise location of an RFID tag is not necessary, less information is needed. Therefore, our system has less computational complexity and less traffic load.

3.4. Other Design Choices. Besides the wireless sensor network operating on the $433 \mathrm{MHz}$ frequency, there are several other types of wireless technologies that are possible candidates to transmit tag data from the client nodes to the server gateway. For example, Crossbow also produces other sensor motes with IEEE 802.15.4/ZigBee ready radio transceivers. Moreover, the wireless communication network can be built using other short-to-medium-distance wireless access technologies such as IEEE 802.15.1/Bluetooth and IEEE 802.11/WLAN. In this section, we give a brief overview of ZigBee, Bluetooth, and WLAN technologies, as well as their limitations in our design application.

Bluetooth and ZigBee are two classes of wireless personal area networks (WPANs) that differ in the data rate, the power consumption, and the quality of service. Since Bluetooth is geared towards handling voice, images, and file transfer, it has a data transfer rate on the order of $1 \mathrm{Mbps}$ with a relatively complex protocol. The operational range for Bluetooth is around 10 meters. With an amplifier antenna, its range can be boosted to 100 meters, but with higher power consumption. ZigBee is designed for systems that need a battery life as long as several months to several years but do not require a data transfer rate as high as those enabled by Bluetooth. The ZigBee-compliant devices have a transmission range between 10 and 75 meters and a data transfer rate of $250 \mathrm{kbps}$. Compared with Bluetooth, ZigBee is more power-efficient because of its small packet size, reduced transceiver duty cycle, reduced complexity, and strict power management mechanisms such as power-down and sleep modes. WLANs allow users in a local area to form a network with high throughput (higher than $1 \mathrm{Mbps}$ ) and reliable data delivery. Compared with WPANs, WLANs have a longer transmission range (around 100 meters) and higher power consumption.

In our system, the client nodes are expected to be batteryoperated and last for a long time without recharge. Bluetooth and WLAN are not selected in our child localization system 


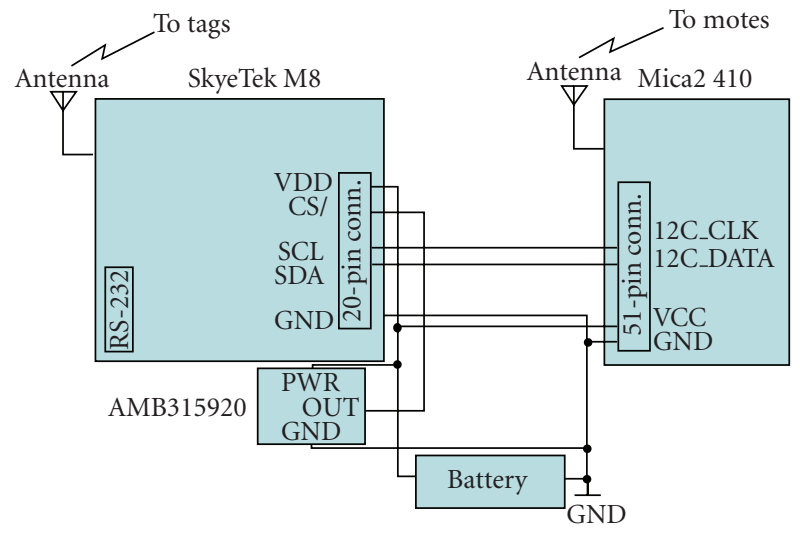

(a) Client node

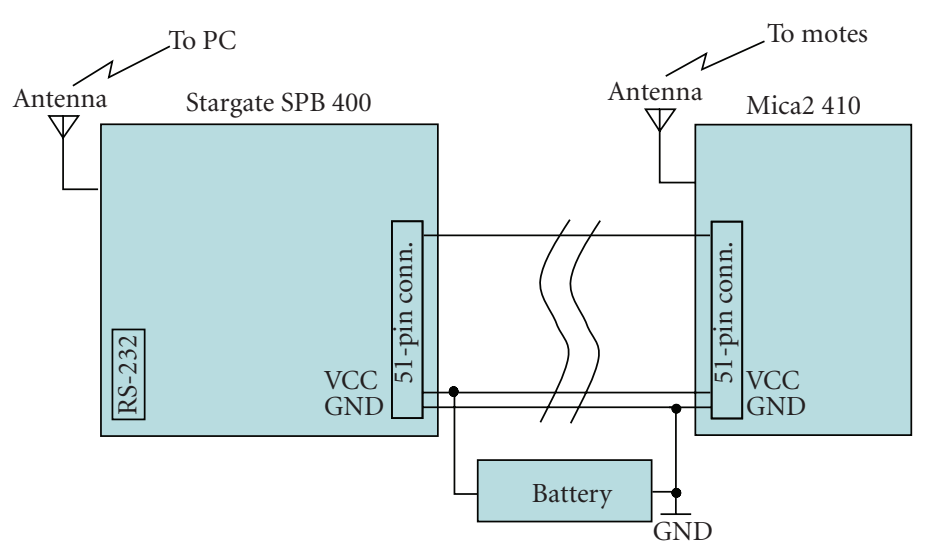

(b) Server gateway

FIgURE 4: System schematics.

because of their higher power consumption. The data transmitted through the wireless communication network are mainly RFID tag UIDs. Therefore, the requirement on the data transfer rate is not high and can be supported by both Crossbow's ZigBee-compliant motes (with $250 \mathrm{kbps}$ data rate) and Mica2 410 motes (with $38.4 \mathrm{kbps}$ data rate). Another consideration in selecting the wireless communication technology is on the operating frequency. Bluetooth, ZigBee, and WLAN are operated on the $2.4 \mathrm{GHz}$ unlicensed industrial, scientific, and medical (ISM) frequency band, which is prone to interference from nearby IEEE 802.11based WLANs. In our system, if the client nodes communicate with each other through a Bluetooth, ZigBee, or WLANbased network, and the Stargate server gateway relays data to a PC base station wirelessly through its $802.11 \mathrm{a} / \mathrm{b}$ card, the back-haul traffic to the PC base station would interfere with the wireless communication among the client nodes. Furthermore, although currently the children's theme park is located in an area without existing wired/wireless network coverage, with the fast deployment and growing coverage of WLAN in the campus, possible interference on the $2.4 \mathrm{GHz}$ frequency band should be taken into consideration. In summary, the Mica2 410 mote is selected in our design mainly because of its low power consumption and its smaller chance of interference from nearby WLANs.

\section{Prototype Testing}

There are three components that need to be tested in our system prototype: the motion detection sensor, the RFID system, and the wireless sensor network.

4.1. Testing the Motion Detection Sensor. The parameter to be tested for the motion detection sensor is the sensitivity or the range of detection. This is essentially the distance that an object has to be placed in order to be detected by the motion detection sensor. We started with placing an object far away from the motion detection sensor where there is no obvious detection. The object was then brought closer to the sensor until the sensitive point where the object was first detected. The detection range was around 1.8 meters, which is close to the marked parameter of the AMB315920 motion detection sensor.

4.2. Testing the RFID System. The transmit power of the RFID reader can be adjusted to achieve different read ranges. The read range ties directly to how precise the localization system will be. If the range is too large, the system cannot give the precise location of the child. If the range is too small, however, the tag needs to be placed very close to the reader, and the reader may fail to identify the child nearby. With the lowest power of $15 \mathrm{~mW}$, the SkyeTek RFID reader has a marked detection range of around 1.5 meters. This range can be increased to 9 meters if the maximum transmit power of $500 \mathrm{~mW}$ is used. We choose the lowest power of $15 \mathrm{~mW}$ so that the RFID reader range is smaller than the range of the motion detection sensor. The tested RFID read range is about 1 meter. The RFID reader also has the capability to detect multiple tags with different UIDs. We used EPC Class 1 Gen 1 and Gen 2 tags with 12 byte UIDs and ISO 18000-6B tags with 8-byte UIDs. Two cases were tested: single tag detection and multiple tags detection with anticollision. In the first case, one tag was placed at about 0.5 meters to the reader. In the second case, different types of tags were mixed and placed at around 0.5 meters to the reader. We tested as many as five tags in the second case. In both cases, the tag(s) can be detected very fast with no discernable delay. Therefore, if multiple children visit a site together, the RFID reader at that site is able to identify them.

4.3. Testing the Wireless Sensor Network. The testing of the wireless sensor network involves the communication range test between a sensor mote and a gateway and the multihop test where a sensor mote can be used as a router to forward another sensor mote's information to the gateway.

We performed the communication tests both indoor and outdoor. The indoor tests were done at the first floor of a classroom building, whereas the outdoor tests were performed in an open parking lot. These tests were done on a Saturday morning when there were little traffic and human disturbance. 
TABLE 2: Range test between a sensor mote and a gateway.

\begin{tabular}{lcc}
\hline & \multicolumn{2}{c}{ Communication range } \\
& Typical (meter) & Maximum (meter) \\
\hline Indoor & 11 & 20 \\
Outdoor & 20 & 25 \\
\hline
\end{tabular}

TABLE 3: Two-hop range test between a sensor mote and a gateway.

\begin{tabular}{lcc}
\hline & \multicolumn{2}{c}{ Two-hop communication range } \\
& Typical (meter) & Maximum (meter) \\
\hline Indoor & 32 & 38 \\
Outdoor & 47 & 52 \\
\hline
\end{tabular}

To test the communication range between two sensor motes, we placed the sensor gateway on a stationary surface. A sensor mote was gradually moved farther away from the fixed gateway at the same surface level until the signal is getting weak. The testing results are listed in Table 2. Under the typical range, the gateway can always receive the signal from the mote without errors. Beyond the typical range, errors start to appear. When the sensor mote is placed at the maximum range, the signal is getting so weak that the gateway almost cannot receive anything.

If the sensor mote can be used as a router to forward other motes' signal to the gateway, the communication range can be extended. We performed the multihop test with another sensor mote added in between the fixed gateway and the previous mote. The new mote acts as a router. With the added sensor mote, the two-hop communication ranges of the indoor and outdoor cases are listed in Table 3. It shows that by adding one router, the communication range can be at least doubled, compared with the results in Table 2. The range can be further extended if more motes in the wireless sensor network can function as routers and the detected data can be transmitted through multiple hops.

4.4. System Test and Traffic Analysis. At the time when the undergraduate design project was carried out, the children's theme park was planned but had not yet been built. Therefore, our tests were performed on a system prototype with one client node (integrated with a motion detection sensor, an RFID reader, and a sensor mote), one sensor mote used as a router, and one server gateway. The system prototype was able to detect multiple tag UIDs once the tags were put close to the client node. The prototype testing results showed that our design is a viable solution to build a child localization system.

The data traffic in the system is affected by the number of children and their mobility pattern in the theme park. With the scale and the size of the planned children's theme park (with fourteen statues of mastodons, i.e., fourteen client nodes), the data traffic volume in the system is not expected to be too high for the following reasons. First, the number of children that are present at the same time in the park is not expected to be too large (possibly well under 100). Second, the data transmitted in the system are limited in size.
For the applications mentioned in Section 3.1, the associated multimedia content can be retrieved from local memory. The data transferred through the system only include the UIDs of the RFID tags, timestamps, and the IDs of the statues. Third, because of the limited moving speed of RFID tags, our application does not require the RFID readers to update tag locations frequently. In an extreme case, if the RFID readers scan for nearby tags and report 12 bytes for RFID tag UID, 8 bytes for timestamp, and 4 bits for statue ID, and if the readers need to update the detection of 100 kids every second, the system data rate is about $16.4 \mathrm{kbps}$, which is well below the RF rate $(38.4 \mathrm{kbps})$ of the sensor mote. Therefore, our system is capable of supporting the data traffic needed for the proposed applications for the children's theme park.

\section{Conclusions and Future Work}

Both RFID and wireless sensor network technologies are employed to build a wireless localization system in a children's theme park. Specifically, we have proposed using passive RFID tagging devices coupled with UHF RFID readers to locate children in the park. The wireless sensor network helps deliver the detection information remotely to a PC base station. Meanwhile, the addition of motion detection sensors further reduces the overall energy consumption of the system. The design and testing experience in this project can enrich the students with the state-of-the-art technologies in a practical application. Moreover, the experience gained from the child localization system can be exported to other applications such as object tracking and surveillance.

After the children's theme park is built and the underlying child localization system is fully implemented, we plan to do more thorough tests on a system with a larger scale. Specifically, with more client nodes added into the system, we plan to test different communication protocols (e.g., suitable MAC and routing protocols) in the wireless sensor network and evaluate their performance. Once the children's theme park is open to the public, we plan to test the tag reading performance when multiple children wearing tags visit a statue together. Moreover, if multimedia content needs to be transmitted over the wireless sensor network to support some advanced activities, and traffic congestion ever occurs at some sensor motes or the gateway, some congestion control algorithms will be investigated.

\section{References}

[1] J. Landt, "The history of RFID," IEEE Potentials, vol. 24, no. 4, pp. 8-11, 2005.

[2] I. F. Akyildiz, W. Su, Y. Sankarasubramaniam, and E. Cayirci, "A survey on sensor networks," IEEE Communications Magazine, vol. 40, no. 8, pp. 102-114, 2002.

[3] K. P. Fishkin, M. Philipose, and A. Rea, "Hands-on RFID: wireless wearables for detecting use of objects," in Proceedings of the International Symposium on Wearable Computers (ISWC '05), pp. 38-43, Osaka, Japan, October 2005.

[4] D. C. Lee and J. Friedman, "Robust object recognition for mobile sensor nets," Tech. Rep. TR-UCLA-NESL-200406-04, Networked \& Embedded Systems Laboratory, University of California, Los Angeles, Calif, USA, 2004. 
[5] "World's largest Wi-Fi location network at LEGOLANDß Denmark," MobileTechNews, 2004, http://www.mobiletechnews.com/info/2004/04/27/011106.html.

[6] C. Swedberg, "Dutch researchers focus on RFID-based sensors for monitoring apnea, epilepsy," RFID Journal, December 2007, http://www.rfidjournal.com/article/articleview/3780.

[7] B. Bacheldor, "Belgium hospital combines RFID, sensors to monitor heart patients," RFID Journal, March 2007, http://www.rfidjournal.com/article/articleview/3120.

[8] B. Bacheldor, "Fighting fires with RFID and wireless sensors," RFID Journal, November 2006, http://www.rfidjournal.com/article/articleview/2799.

[9] "Sprinkling RFID sensor tags from the sky," RFID in Japan, March 2006, http://rfidinjapan.wordpress.com/2006/03/20/ sprinkling-rfid-sensor-tags-from-the-sky/.

[10] M. Roberti, "Navy revs up RFID sensors," RFID Journal, June 2004, http://www.rfidjournal.com/article/articleview/990.

[11] R. Wessel, "Cargo-tracking system combines RFID, sensors, GSM and satellite," RFID Journal, January 2008, http://www.rfidjournal.com/article/articleview/3870.

[12] C. Swedberg, "ZigBeef offers ranchers a long-distance cattle head count," RFID Journal, February 2008, http://www .rfidjournal.com/article/articleview/3935.

[13] C. Chen, C. Aneke, J. Ebaugh, and C. Hong, "Development of a child localization system on RFID and sensor networks in an undergraduate capstone senior design project," in Proceedings of ASEE Illinois-Indiana and North Central Conference, Fort Wayne, Ind, USA, March-April 2006.

[14] L. Ho, M. Moh, Z. Walker, T. Hamada, and C.-F. Su, "A prototype on RFID and sensor networks for elder healthcare: progress report," in Proceedings of ACM Workshop on Experimental Approaches to Wireless Network Design and Analysis (EWIND), pp. 70-75, Philadelphia, Pa, USA, August 2005.

[15] "UHF vs. HF RFID: new insight on the old debate," Intermec Inc., 2007, http://www.rfidproductnews.com/whitepapers/files/0702_uhf_vs_hfrfid.pdf.

[16] "SkyeRead M8 reference guide (document version 1.6)," SkyeTek Inc., 2005, http://support.skyetek.com/.

[17] "SkyeTek protocol v.2.2," SkyeTek Inc., 2004, http://support .skyetck.com/.

[18] "MPR/MIB mote hardware users manual (document 74300021-07)," Crossbow Inc., 2005, http://www.xbow.com/Support/wUserManuals.aspx.

[19] "Stargate developer's guide (document 7430-0317-13)," Crossbow Inc., 2005, http://www.xbow.com/Support/wUserManuals.aspx.

[20] "MA motion sensor (area reflective type)," Panasonic Inc., http://pewa.panasonic.com/pcsd/product/sens/pdf_cat/amb .pdf. 

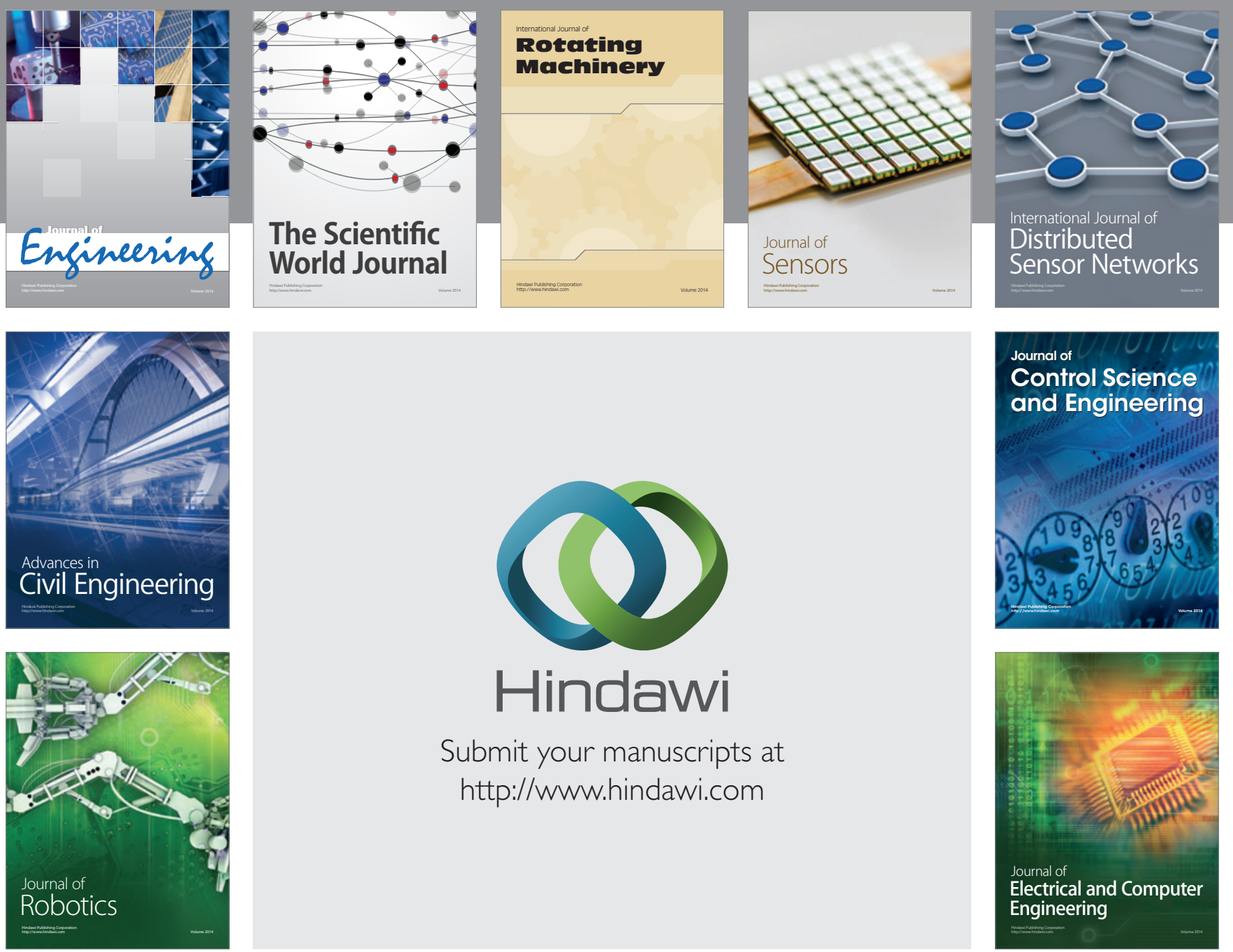

Submit your manuscripts at

http://www.hindawi.com
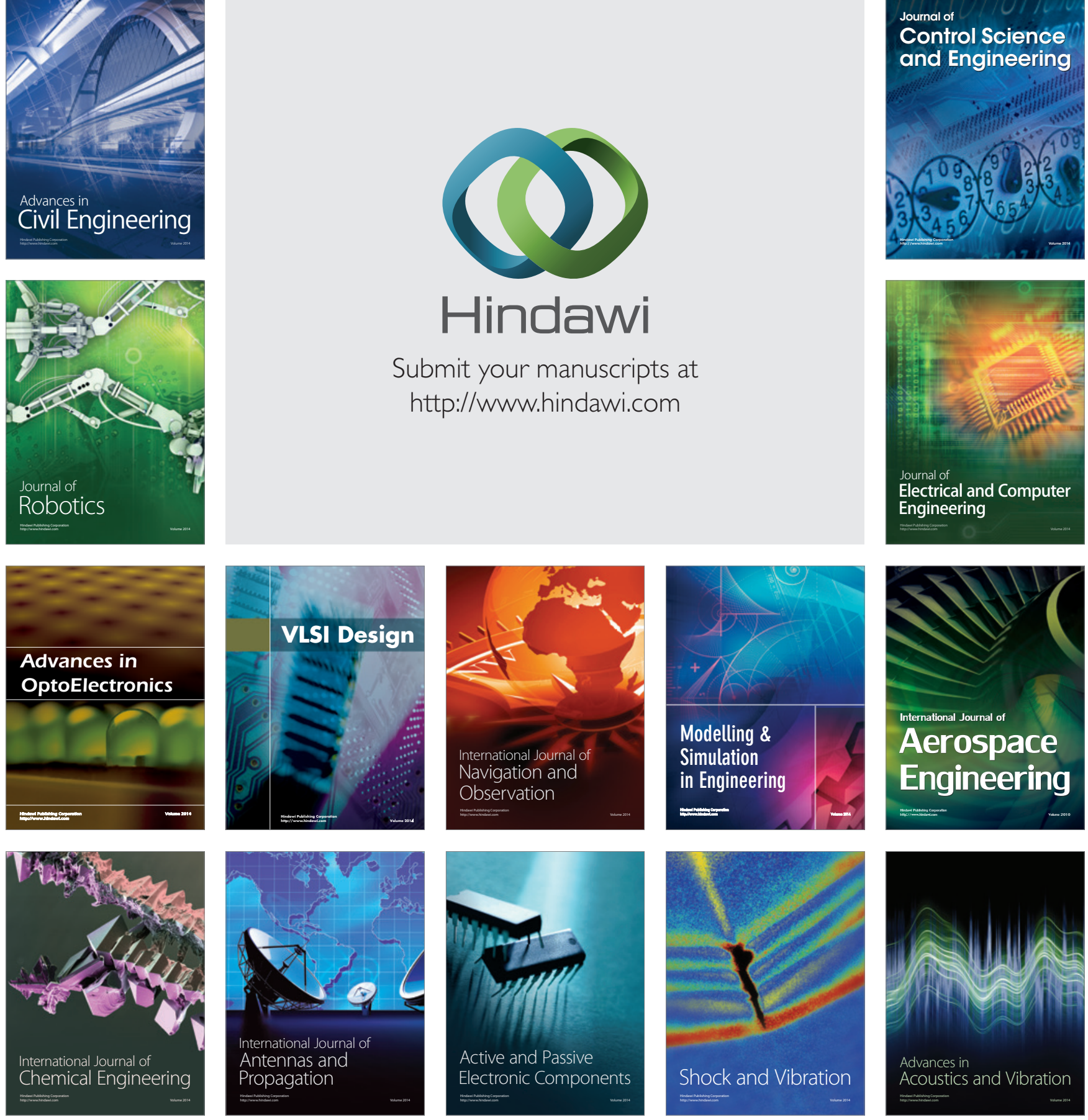